\title{
A Passivity Criterion for N-Port Multilateral Haptic Systems
}

\author{
Victor Mendez and Mahdi Tavakoli
}

\begin{abstract}
This paper presents a criterion for passivity of $n$ port networks, which can be used to model multilateral systems involving haptic information sharing between a number of users. Such systems have recently found interesting applications in both cooperative haptic teleoperation and haptics-assisted training. The criterion presented in Theorem 1, which is necessary and sufficient for passivity of the $n$-port network, imposes $2 n$ conditions on the immitance parameters of the network and on the residues of the immitance parameters at their imaginary-axis poles. It is shown that when $n=2$, the proposed conditions reduce to the well-known Raisbeck's passivity criterion for two-port networks. Another special case for which the proposed criterion has been simplified corresponds to three-port networks. Finally, the passivity of a dual-user haptic system for control of a single teleoperated robot is investigated.
\end{abstract}

\section{INTRODUCTION}

Two-port networks are overwhelmingly the method of choice for modeling a bilateral teleoperation system, which consists of a slave robot and a master user interface. The human operator controls the slave and is provided with haptic feedback concerning slave/environment contact forces through the master. Fig.1 shows the equivalent circuit representation of a teleoperation system. Usually, only the linear dynamics of the master and slave are considered:

$f_{m}+f_{h}=M_{m} \ddot{x}_{m}, \quad f_{s}-f_{e}=M_{s} \ddot{x}_{s}$

where the hand/master interaction is denoted by $f_{h}$ and the slave/environment interaction is denoted by $f_{e} . M_{m}, M_{s}, x_{m}, x_{s}$, $f_{m}$, and $f_{s}$ are the master and slave inertias, positions, and control signals, respectively. Impedances $Z_{h}$ and $Z_{e}$ denote the dynamics characteristics of the human operator's hand and the remote environment, respectively. $F_{h}{ }^{*}$ and $F_{e}{ }^{*}$ are the operator's and environment's exogenous input forces, which are independent of the teleoperation system behavior [1].

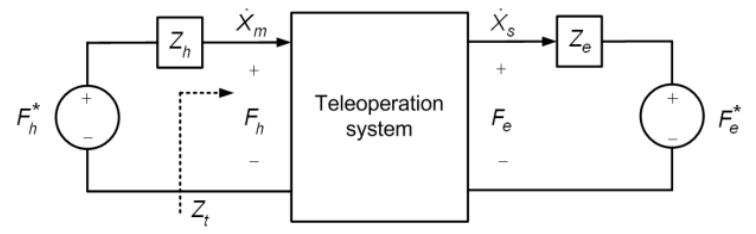

Fig. 1: Two-port model of a bilateral teleoperation system

Fig. 2 shows a general 2-port network. Depending on which combination of these four quantities $\left(I_{1}, I_{2}, V_{l}, V_{2}\right)$ are chosen as independent and dependent variable pairs, six different ways for modeling the 2-port network exist. For instance, using the impedance parameters, the 2-port network can be modeled as

\footnotetext{
${ }^{1}$ This research was supported by the Natural Sciences and Engineering Research Council (NSERC) of Canada under grants RGPIN-372042 and EQPEQ-375712. The authors are with the Department of Electrical and Computer Engineering, University of Alberta, Edmonton, Alberta, T6G 2V4 Canada. (e-mail: vmendez@ualberta.ca, tavakoli@ece.ualberta.ca).
}

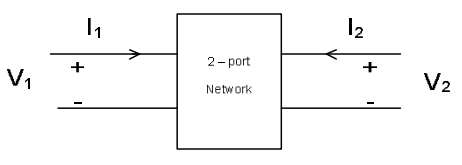

Fig. 2: A general 2-port network

$$
\left[\begin{array}{l}
V_{1} \\
V_{2}
\end{array}\right]=\left[\begin{array}{ll}
z_{11} & z_{12} \\
z_{21} & z_{22}
\end{array}\right]\left[\begin{array}{l}
I_{1} \\
I_{2}
\end{array}\right]
$$

Accordingly, the impedance model of the bilateral teleoperation system in Fig. 1 is given by

$$
\left[\begin{array}{l}
F_{h} \\
F_{e}
\end{array}\right]=\left[\begin{array}{ll}
z_{11} & z_{12} \\
z_{21} & z_{22}
\end{array}\right]\left[\begin{array}{c}
\dot{X}_{m} \\
-\dot{X}_{s}
\end{array}\right]
$$

The main goals of teleoperation control are transparency and stability. Transparency is the ability of a teleoperation system to present the undistorted dynamics of the remote environment to the human operator [2], and requires the master and the slave positions and interactions to match regardless of the operator and environment dynamics

$f_{h}=f_{e}, \quad x_{m}=x_{s}$

Taking precedence to transparency is closed-loop stability, which is crucial for safe teleoperation. For the analysis of closed-loop stability of a teleoperation system, according to Fig. 1, the knowledge of the human operator and the environment dynamics are needed in addition to the teleoperation system immitance parameters $(z, y, h$, or $g)$. In practice, however, the model for the human operator and environment are usually unknown, uncertain, and/or timevarying. In fact, the dynamic parameters of the human operator change in response to the specific requirements of the task at hand [3], [4], and the dynamic parameters of an environment may also change over time. This makes it impossible to use conventional techniques to investigate the closed-loop stability of a teleoperation system. However, assuming that $Z_{h}(s)$ and $Z_{e}(s)$ in Fig. 1 are passive, we might be able to draw stability conditions that are independent of the human operator and the environment (see Section II).

On the other hand, some tasks can be performed more effectively using two hands rather than one or through collaboration rather than individual operation. Also, by using multiple master interfaces each with a corresponding slave robot, multilateral tele-cooperation systems enable haptic information sharing and collaboration in performing a task in a remote environment by multiple users [5], [6], [7], [8], [9]. The key difference between a multilateral tele-cooperation system and a bilateral teleoperation (i.e., single-master/singleslave) system is that the former cannot be modeled as a 2-port network. Therefore, conventional theories for absolute stability or passivity analysis of 2-port networks will not be adequate for multilateral haptic systems. Therefore, there is a need for tools that can analyze the passivity of multi-user haptic systems modeled as $n$-port networks. The only tool available to date for checking the passivity of an $n$-port is 
based on the singular value of the scattering matrix of the network [10]:

$$
\|S\|_{\infty}=\bar{\sigma}\left(S^{T}(j \omega) S(j \omega)\right)
$$

The above condition is difficult to verify in the general case and without knowledge of the model parameter values for the robots and the controllers (making it not suitable for control synthesis). The criterion we propose in Section III is necessary and sufficient for passivity of the $n$-port network and is easy to check as it imposes $2 n$ conditions directly on the immitance parameters of the network and on the residues of the immitance parameters at their imaginary-axis poles.

\section{Absolute Stability and Passivity for 2-Port NETWORKS}

Two well-known methods have been developed to investigate the stability of a 2-port network connected to unknown terminations. These methods are known as Llewellyn's absolute stability criterion and Raisbeck's passivity criterion. Both criteria work under the assumption that the operator and the environment are passive. By definition, a 2-port network is absolutely stable if it remains stable under all possible uncoupled passive terminations. Also by definition, a 2-port network is passive if the total energy delivered to the network at its input and output ports is non-negative [11].

\section{A. Llewellyn's absolute stability criterion:}

If $p$ represents any of the four immitance parameters $(z, y, h$, $g$ ) of a 2-port network, the criterion establishes that the network is absolutely stable if and only if [11]

- $\quad p_{11}$ and $p_{22}$ have no poles in the right-half plane (RHP)

- Any poles of $p_{11}$ and $p_{22}$ on the imaginary axis are simple with real and positive residues

- For all real values of frequencies $\omega$, we have

$\operatorname{Re} p_{11} \geq 0$

$\operatorname{Re} p_{22} \geq 0$

$2 \operatorname{Re} p_{11} \operatorname{Re} p_{22}-\operatorname{Re}\left(p_{12} p_{21}\right)-\left|p_{12} p_{21}\right| \geq 0$

where $\operatorname{Re}$ denotes the real part.

B. Raisbeck's passivity criterion:

The necessary and sufficient conditions for passivity of a 2port network with the immitance parameter $p$ are [11]

- The $p$-parameters have no RHP poles.

- Any poles of the $p$-parameters on the imaginary axis are simple, and the residues of the $p$-parameters at these poles satisfy the following conditions:

$\circ$ If $k_{i j}$ denotes the residue of $p_{i j}$ and $k_{i j}^{*}$ is the complex conjugate of $k_{j i}$, then

$$
\begin{aligned}
& k_{11} \geq 0 \\
& k_{22} \geq 0 \\
& k_{11} k_{22}-k_{12} k_{21} \geq 0, \text { with } k_{21}=k_{12}^{*}
\end{aligned}
$$

- The real and imaginary part of the $p$-parameters satisfy the following conditions for all real frequencies $\omega$

$$
\begin{aligned}
& \operatorname{Re} p_{11} \geq 0 \\
& \operatorname{Re} p_{22} \geq 0 \\
& 4 \operatorname{Re} p_{11} \operatorname{Re} p_{22}-\left(\operatorname{Re} p_{12}+\operatorname{Re} p_{21}\right)^{2}-\left(\operatorname{Im} p_{12}-\operatorname{Im} p_{21}\right)^{2} \geq 0
\end{aligned}
$$

where Im denotes the imaginary part.

So far, these criteria have not been extended to $n$-port networks where $n>2$. Our objective is to give necessary and sufficient conditions for passivity of a general $n$-port network, which can represent a system where multiple haptic teleoperators are used to perform a physical or virtual task collaboratively. Nonetheless, the proposed criterion can be applied for passivity analysis of any $n$-port network including microwave circuits [12], [13].

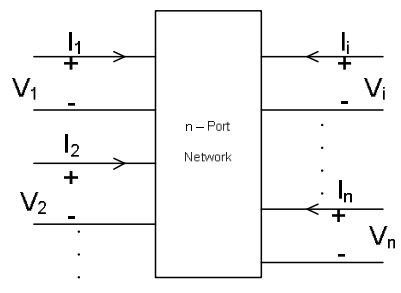

Fig. 3: A general $n$-port network

\section{Main Result: Passivity Conditions for N-Port NETWORKS}

An $n$-port network can be defined as a network containing $n$ pairs of terminals for external connections. Each pair of terminals represents a port to which an external network can be connected (Fig. 3). The external behavior of the $n$-port network can be determined if all the $I_{i}$ currents and $V_{i}$ voltages are known. In this section, we present the necessary and sufficient conditions for passivity of an $n$-port network. By analogy with 2-port networks, an $n$-port network is passive if, for all excitations, the total energy delivered to the network at its input and output ports is non-negative. Mathematically, this passivity definition is expressed as

$E(t)=\int_{-\infty}^{t}\left(v_{1}(\tau) i_{1}(\tau)+v_{2}(\tau) i_{2}(\tau)+\ldots+v_{n}(\tau) i_{n}(\tau)\right) d \tau \geq 0$

where $E(t)$ is the total energy delivered to the $n$-port network. We know that the passivity of a 1-port network is a necessary and sufficient condition for positive-realness of its impedance, which is equivalent to

$\operatorname{Re}\left(V(s) I^{*}(s)\right) \geq 0$ for $\operatorname{Re} s \geq 0$

By analogy, (9) is equivalent to the following condition

$\operatorname{Re}\left(V_{1}(s) I_{1}^{*}(s)+\ldots+V_{n}(s) I_{n}^{*}(s)\right) \geq 0$ for $\operatorname{Re} s \geq 0$

where $I_{i}^{*}(s)$ is the complex conjugate of $I_{i}(s)$.

Using impedance parameters of the $n$-port network, the relations between voltages and currents are

$\left[\begin{array}{c}V_{1}(s) \\ V_{2}(s) \\ \vdots \\ V_{n}(s)\end{array}\right]=\left[\begin{array}{cccc}z_{11}(s) & z_{12}(s) & \cdots & z_{1 n}(s) \\ z_{21}(s) & z_{22}(s) & \cdots & z_{2 n}(s) \\ \vdots & \vdots & \ddots & \vdots \\ z_{n 1}(s) & z_{n 2}(s) & \cdots & z_{n n}(s)\end{array}\right]\left[\begin{array}{c}I_{1}(s) \\ I_{2}(s) \\ \vdots \\ I_{n}(s)\end{array}\right]$

which can be compactly described as $\mathbf{V}=\mathbf{Z I}$. The following Theorem holds for any of the four immittance parameters, yet for brevity we write it only in terms of impedance parameters. Theorem 1: The necessary and sufficient conditions for passivity (defined by (11)) of an $n$-port network are:

- The $p$-parameters have no RHP poles. 
- Any poles of the $p$-parameters on the imaginary axis are simple, and the residues of the $p$-parameters at these poles satisfy the following conditions:

$$
\begin{aligned}
& \text { 1. } k_{i i} \geq 0, \quad i=1, \cdots, n \\
& \text { 2. } \frac{k_{11} k_{22}-k_{12} k_{21}}{k_{11}} \geq 0 \\
& \text { 3. } \frac{k_{11} k_{33}-k_{13} k_{31}}{k_{11}}-\frac{\left(k_{11} k_{23}-k_{21} k_{13}\right)}{\left(k_{11} k_{22}-k_{12} k_{21}\right)} \frac{\left(k_{11} k_{32}-k_{31} k_{12}\right)}{k_{11}} \geq 0 \\
& \vdots \\
& \text { n. } k_{n n}-\sum_{i=1}^{n-1}\left|u_{i n}\right|^{2} k_{i i}^{\prime} \geq 0
\end{aligned}
$$

where $k_{i j}$ denotes the residue of $p_{i j}$ and $k_{i j}^{*}$ is the complex conjugate of $k_{j i}$. The terms $u_{i j}$ are the elements of an upper triangular matrix $\mathbf{U}$ used to diagonalize the matrix $\mathbf{K}$ according to $\mathbf{U}^{*} \mathbf{K}^{\prime} \mathbf{U}=\mathbf{K}$. The coefficients $k_{i i}^{\prime}$ are the elements of the diagonal matrix $\mathbf{K}^{\prime}$.

- The real and imaginary parts of the $z$-parameters satisfy the following conditions for all real frequencies $\omega$

$$
\begin{aligned}
& \text { 1. } z_{11}^{\prime} \geq 0 \\
& \text { 2. } \frac{z_{11}^{\prime} z_{22}^{\prime}-z_{12}^{\prime} z_{21}^{\prime}}{z_{11}^{\prime}} \geq 0 \\
& \text { 3. } \frac{z_{11}^{\prime} z_{33}^{\prime}-z_{13}^{\prime} z_{31}^{\prime}}{z_{11}^{\prime}}-\frac{\left(z_{11}^{\prime} z_{23}^{\prime}-z_{21}^{\prime} z_{13}^{\prime}\right)}{\left(z_{11}^{\prime} z_{22}^{\prime}-z_{12}^{\prime} z_{21}^{\prime}\right)} \frac{\left(z_{11}^{\prime} z_{32}^{\prime}-z_{31}^{\prime} z_{12}^{\prime}\right)}{z_{11}^{\prime}} \geq 0 \\
& \quad \vdots \\
& \text { n. } z_{n n}^{\prime}-\sum_{i=1}^{n-1}\left|w_{i n}\right|^{2} z_{i i}^{\prime} \geq 0
\end{aligned}
$$

where $z_{i j}^{\prime}=\frac{1}{2}\left(z_{i j}+z_{j i}^{*}\right)$. The terms $w_{i j}$ are the elements of an upper triangular matrix $\mathbf{W}$ used to diagonalize the matrix $\mathbf{Z}^{\prime}$ according to $\mathbf{Z}^{\prime}=\mathbf{W}^{*} \mathbf{Z}^{\prime \prime} \mathbf{W}$. The elements $z_{i i}^{\prime \prime}$ are the entries of the diagonal matrix $\mathbf{Z}^{\prime \prime}$.

Proof: Eliminating the voltages in (11) by using (12), we find that the $n$-port network passivity is equivalent to

$\operatorname{Re}[F(s)] \geq 0$ for $\operatorname{Re} s \geq 0$

where

$$
\begin{aligned}
\operatorname{Re} F(s)=\operatorname{Re} & {\left[z_{11}(s) I_{1}(s) I_{1}^{*}(s)+\cdots+z_{1 n}(s) I_{n}(s) I_{1}^{*}(s)\right.} \\
& +z_{21}(s) I_{1}(s) I_{2}^{*}(s)+\cdots+z_{2 n}(s) I_{n}(s) I_{2}^{*}(s) \\
& +\cdots+ \\
& \left.+z_{n 1}(s) I_{1}(s) I_{n}^{*}(s)+\cdots+z_{n n}(s) I_{n}(s) I_{n}^{*}(s)\right]
\end{aligned}
$$

.

condition $\mathrm{B}$, assume that $F(s)$ has a simple pole at $s=j \omega_{0}$ with a residue $k_{0}$. Let $k_{11}, k_{12} \cdots k_{21}, \cdots k_{n n}$ denote the residues of $z_{11}, z_{12} \cdots z_{21} \cdots z_{n n}$, respectively, at this pole. Expanding $F(s)$ in a Laurent series about $s=j \omega_{0}$ and keeping only the dominant terms in the neighborhood of the pole, we get

$$
\begin{aligned}
& \frac{k_{0}}{s-j \omega_{0}}= \\
& \frac{k_{11}\left(j \omega_{0}\right) I_{1}\left(j \omega_{0}\right) I_{1}^{*}\left(j \omega_{0}\right)}{s-j \omega_{0}}+\cdots+\frac{k_{1 n}\left(j \omega_{0}\right) I_{n}\left(j \omega_{0}\right) I_{1}^{*}\left(j \omega_{0}\right)}{s-j \omega_{0}}+\cdots \\
& +\frac{k_{n 1}\left(j \omega_{0}\right) I_{1}\left(j \omega_{0}\right) I_{n}^{*}\left(j \omega_{0}\right)}{s-j \omega_{0}}+\cdots+\frac{k_{n n}\left(j \omega_{0}\right) I_{n}\left(j \omega_{0}\right) I_{n}^{*}\left(j \omega_{0}\right)}{s-j \omega_{0}}
\end{aligned}
$$

which is equivalent to

$k_{0}=$

$$
\begin{aligned}
& k_{11}\left(j \omega_{0}\right) I_{1}\left(j \omega_{0}\right) I_{1}^{*}\left(j \omega_{0}\right)+\cdots+k_{1 n}\left(j \omega_{0}\right) I_{n}\left(j \omega_{0}\right) I_{1}^{*}\left(j \omega_{0}\right)+\cdots \\
& +k_{n 1}\left(j \omega_{0}\right) I_{1}\left(j \omega_{0}\right) I_{n}^{*}\left(j \omega_{0}\right)+\cdots+k_{n n}\left(j \omega_{0}\right) I_{n}\left(j \omega_{0}\right) I_{n}^{*}\left(j \omega_{0}\right)
\end{aligned}
$$

In (18), $k_{0}$ must be a real and non-negative number to satisfy condition $\mathrm{B}$. All the $k_{i i}$ for $i=1, \cdots, n$ are real and positive since the impedances $z_{i i}(s)$ are positive real functions. Also, $I_{i}\left(j \omega_{0}\right) I_{i}^{*}\left(j \omega_{0}\right)$ is real and positive. Note that in the pairs $k_{i j}\left(j \omega_{0}\right) I_{j}\left(j \omega_{0}\right) I_{i}^{*}\left(j \omega_{0}\right)+k_{j i}\left(j \omega_{0}\right) I_{i}\left(j \omega_{0}\right) I_{j}^{*}\left(j \omega_{0}\right), \quad$ since $I_{j}\left(j \omega_{0}\right) I_{i}^{*}\left(j \omega_{0}\right)$ and $I_{i}\left(j \omega_{0}\right) I_{j}^{*}\left(j \omega_{0}\right)$ are complex conjugates, $k_{i j}$ and $k_{j i}$ are also complex conjugates.

Since the right side of (18) is a Hermitian form, it can be diagonalized. To do so, (18) can be written in matrix form as

$$
k_{0}=\left[\begin{array}{llll}
I_{1}^{*} & I_{2}^{*} & \cdots & I_{n}^{*}
\end{array}\right]\left[\begin{array}{cccc}
k_{11} & k_{12} & \cdots & k_{1 n} \\
k_{21} & k_{22} & \cdots & k_{2 n} \\
\vdots & \vdots & \ddots & \vdots \\
k_{n 1} & k_{n 2} & \cdots & k_{n n}
\end{array}\right]\left[\begin{array}{c}
I_{1} \\
I_{2} \\
\vdots \\
I_{n}
\end{array}\right]=\mathbf{I}^{*} \mathbf{K} \mathbf{I}
$$

The K-matrix is diagonalizable and we want to find a linear transformation such as $\mathbf{U}^{*} \mathbf{K}^{\prime} \mathbf{U}=\mathbf{K}$ where $\mathbf{K}^{\prime}$ is a diagonal matrix, $\mathbf{U}$ is an upper triangular matrix, and $\mathbf{U}^{*}$ (the transpose complex conjugate of $\mathbf{U}$ ) is a lower triangular matrix. By representing the $\mathbf{U}$ matrix in the reduced rowechelon form, we arrive to the following system

$$
\begin{aligned}
& {\left[\begin{array}{ccccc}
1 & 0 & 0 & \cdots & 0 \\
u_{12}^{*} & 1 & 0 & \cdots & 0 \\
u_{13}^{*} & u_{23}^{*} & 1 & \ddots & 0 \\
\vdots & \vdots & \ddots & \ddots & \vdots \\
u_{1 n}^{*} & u_{2 n}^{*} & u_{3 n}^{*} & \cdots & 1
\end{array}\right] \cdot\left[\begin{array}{ccccc}
k_{11}^{\prime} & 0 & 0 & \cdots & 0 \\
0 & k_{22}^{\prime} & 0 & \cdots & 0 \\
0 & 0 & k_{33}^{\prime} & \ddots & 0 \\
\vdots & \vdots & \ddots & \ddots & \vdots \\
0 & 0 & 0 & \cdots & k_{n n}^{\prime}
\end{array}\right] .} \\
& {\left[\begin{array}{ccccc}
1 & u_{12} & u_{13} & \cdots & u_{1 n} \\
0 & 1 & u_{23} & \cdots & u_{2 n} \\
0 & 0 & 1 & \ddots & u_{3 n} \\
\vdots & \vdots & \vdots & \ddots & \vdots \\
0 & 0 & 0 & \cdots & 1
\end{array}\right]=\left[\begin{array}{ccccc}
k_{11} & k_{12} & k_{13} & \cdots & k_{1 n} \\
k_{21} & k_{22} & k_{23} & \cdots & k_{2 n} \\
k_{31} & k_{32} & k_{33} & \cdots & k_{3 n} \\
\vdots & \vdots & \vdots & \ddots & \vdots \\
k_{n 1} & k_{n 2} & k_{n 3} & \cdots & k_{n n}
\end{array}\right]}
\end{aligned}
$$
$F(s)$ meets the following conditions:

A. $\quad F(s)$ has no RHP poles

B. Any poles of $F(s)$ on the imaginary axis are simple with real and non-negative residues

C. $\quad \operatorname{Re}[F(j \omega)] \geq 0, \quad \forall \omega$

For condition $\mathrm{A}$, we require that none of the $z$-parameters of the $n$-port network have any poles in the RHP. For
Solving for $\mathbf{K}^{\prime}$ and $\mathbf{U}$ will lead us to expressions for each $k_{i i}^{\prime}$ as a function of $k_{i j}$ elements. The left side of (20) is 


$$
\left[\begin{array}{ccc}
k_{11}^{\prime} & k_{11}^{\prime} u_{12} & k_{11}^{\prime} u_{13} \\
u_{12}^{*} k_{11}^{\prime} & u_{12}^{*} k_{11}^{\prime} u_{12}+k_{22}^{\prime} & u_{12}^{*} k_{11}^{\prime} u_{13} \\
u_{13}^{*} k_{11}^{\prime} & u_{13}^{*} k_{11}^{\prime} u_{12}+u_{23}^{*} k_{22}^{*} & u_{13}^{*} k_{11}^{\prime} u_{13}+u_{23}^{*} k_{22}^{\prime} u_{23}+k_{33}^{\prime} \\
\vdots & \vdots & \vdots \\
u_{1 n}^{*} k_{11}^{\prime} & u_{1 n}^{*} k_{11}^{\prime} u_{12}+u_{2 n}^{*} k_{22}^{\prime} & u_{1 n}^{*} k_{11}^{\prime} u_{13}^{\prime}+u_{2 n}^{*} k_{22}^{\prime} u_{23}+u_{3 n}^{\prime} k_{33}^{\prime} \\
\ldots & k_{11}^{\prime} u_{1 n} \\
\cdots & u_{12}^{*} k_{11}^{\prime} u_{1 n}+k_{22}^{\prime} u_{2 n} \\
\cdots & u_{13}^{*} k_{11}^{\prime} u_{1 n}+u_{23}^{*} k_{22}^{\prime} u_{2 n}+k_{33}^{\prime} u_{3 n} \\
\ddots & \vdots \\
\cdots & \sum_{i=1}^{n}\left|u_{i n}\right|^{2} k_{i i}^{\prime}
\end{array}\right]
$$

The solution to (20) is

$$
\begin{aligned}
& k_{11}^{\prime}=k_{11} \\
& k_{22}^{\prime}=\frac{k_{11} k_{22}-k_{12} k_{21}}{k_{11}} \\
& k_{33}^{\prime}=\frac{k_{11} k_{33}-k_{13} k_{31}}{k_{11}}-\frac{\left(k_{11} k_{23}-k_{21} k_{13}\right)}{\left(k_{11} k_{22}-k_{12} k_{21}\right)} \frac{\left(k_{11} k_{32}-k_{31} k_{12}\right)}{k_{11}} \\
& \vdots \\
& k_{n n}^{\prime}=k_{n n}-\sum_{i=1}^{n-1}\left|u_{i n}\right|^{2} k_{i i}^{\prime}
\end{aligned}
$$

Now, (19) can be rewritten as

$$
k_{0}=\mathbf{I}^{*} \mathbf{K} \mathbf{I}=\mathbf{I}^{*} \mathbf{U}^{*} \mathbf{K}^{\prime} \mathbf{U I}=(\mathbf{U I})^{*} \mathbf{K}^{\prime}(\mathbf{U I})
$$

implying that $k_{0}$ will be non-negative and equivalently condition B holds iff $k_{i i}^{\prime}$ in (22) are all non-negative (this also implies $\left.k_{22} \geq 0, k_{33} \geq 0, k_{44} \geq 0, \cdots k_{n n} \geq 0\right)$. Therefore, it is established that condition B holds iff (13) holds.

Regarding condition $\mathrm{C}$, the real part of $F(j \omega)$ can be obtained from $\operatorname{Re}[F(j \omega)]=\frac{1}{2}\left[F(j \omega)+F^{*}(j \omega)\right]$. By using $z_{i j}^{\prime}=\frac{1}{2}\left(z_{i j}+z_{j i}^{*}\right)$, the real part of $F(j \omega)$ can be written as $\operatorname{Re}[F(j \omega)]=$

$$
\begin{aligned}
& z_{11}^{\prime}(j \omega) I_{1}(j \omega) I_{1}^{*}(j \omega)+\cdots+z_{1 n}^{\prime}(j \omega) I_{n}(j \omega) I_{1}^{*}(j \omega)+\cdots+ \\
& \left.z_{n 1}^{\prime}(j \omega) I_{1}(j \omega) I_{n}^{*}(j \omega)+\cdots+z_{n n}^{\prime}(j \omega) I_{n}(j \omega) I_{n}^{*}(j \omega)\right] \\
& \text { or equivalently as } \\
& \operatorname{Re}[F(j \omega)]=\mathbf{I}^{*} \mathbf{Z}^{\prime} \mathbf{I}
\end{aligned}
$$

where

$$
\begin{aligned}
& \mathbf{Z}^{\prime}=\left[\begin{array}{cccc}
z_{11}^{\prime}(j \omega) & z_{12}^{\prime}(j \omega) & \cdots & z_{1 n}^{\prime}(j \omega) \\
z_{21}^{\prime}(j \omega) & z_{22}^{\prime}(j \omega) & \cdots & z_{2 n}^{\prime}(j \omega) \\
\vdots & \vdots & \ddots & \vdots \\
z_{n 1}^{\prime}(j \omega) & z_{n 2}^{\prime}(j \omega) & \cdots & z_{n n}^{\prime}(j \omega)
\end{array}\right]=
\end{aligned}
$$

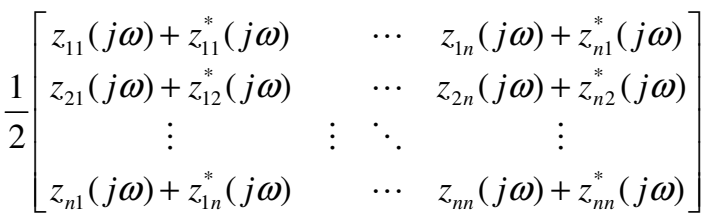

The $z$-parameters have complex values, i.e., $z_{i j}=r_{i j}+$ $j x_{i j}$ where $r_{i j}$ is the real part and $x_{i j}$ is the imaginary part of $z_{i j}$.It is easy to see that (24) is a Hermitian form. Using a procedure similar to (19)-(23), which was for the residue matrix, $\mathbf{Z}^{\prime}$ can be expressed as $\mathbf{Z}^{\prime}=\mathbf{W}^{*} \mathbf{Z}^{\prime \prime} \mathbf{W}$ where $\mathbf{Z}^{\prime \prime}$ is a diagonal matrix and $\mathbf{W}$ is an upper triangular matrix.

$\left[\begin{array}{ccccc}1 & 0 & 0 & \cdots & 0 \\ w_{12}^{*} & 1 & 0 & \cdots & 0 \\ w_{13}^{*} & w_{23}^{*} & 1 & \ddots & 0 \\ \vdots & \vdots & \ddots & \ddots & \vdots \\ w_{1 n}^{*} & w_{2 n}^{*} & w_{3 n}^{*} & \cdots & 1\end{array}\right]\left[\begin{array}{ccccc}z_{11}^{\prime \prime} & 0 & 0 & \cdots & 0 \\ 0 & z_{22}^{\prime \prime} & 0 & \cdots & 0 \\ 0 & 0 & z_{33}^{\prime \prime} & \ddots & 0 \\ \vdots & \vdots & \ddots & \ddots & \vdots \\ 0 & 0 & 0 & \cdots & z_{n n}^{\prime \prime}\end{array}\right]$
$\left[\begin{array}{ccccc}1 & w_{12} & w_{13} & \cdots & w_{1 n} \\ 0 & 1 & w_{23} & \cdots & w_{2 n} \\ 0 & 0 & 1 & \ddots & w_{3 n} \\ \vdots & \vdots & \vdots & \ddots & \vdots \\ 0 & 0 & 0 & \cdots & 1\end{array}\right]=\left[\begin{array}{ccccc}z_{11}^{\prime} & z_{12}^{\prime} & z_{13}^{\prime} & \cdots & z_{1 n}^{\prime} \\ z_{21}^{\prime} & z_{22}^{\prime} & z_{23}^{\prime} & \cdots & z_{2 n}^{\prime} \\ z_{31}^{\prime} & z_{32}^{\prime} & z_{33}^{\prime} & \cdots & z_{3 n}^{\prime} \\ \vdots & \vdots & \vdots & \ddots & \vdots \\ \vdots & \vdots & \vdots & \cdots & \vdots \\ z_{n 1} & z_{n 2} & z_{n 3}^{\prime} & \cdots & z_{n n}^{\prime}\end{array}\right]$

The solution to (27) is

$$
\begin{aligned}
& z_{11}^{\prime \prime}=z_{11}^{\prime} \\
& z_{22}^{\prime \prime}=\frac{z_{11}^{\prime} z_{22}^{\prime}-z_{12}^{\prime} z_{21}^{\prime}}{z_{11}^{\prime}} \\
& z_{33}^{\prime \prime}=\frac{z_{11}^{\prime} z_{33}^{\prime}-z_{13}^{\prime} z_{31}^{\prime}}{z_{11}^{\prime}}-\frac{\left(z_{11}^{\prime} z_{23}^{\prime}-z_{21}^{\prime} z_{13}^{\prime}\right)}{\left(z_{11}^{\prime} z_{22}^{\prime}-z_{12}^{\prime} z_{21}^{\prime}\right)} \frac{\left(z_{11}^{\prime} z_{32}^{\prime}-z_{31}^{\prime} z_{12}^{\prime}\right)}{z_{11}^{\prime}} \\
& \vdots \\
& z_{n n}^{\prime \prime}=z_{n n}^{\prime}-\sum_{i=1}^{n-1}\left|w_{i n}\right|^{2} z_{i i}^{\prime}
\end{aligned}
$$

Now, (25) can be rewritten as

$$
\operatorname{Re}[F(j \omega)]=\mathbf{I}^{*} \mathbf{Z}^{\prime} \mathbf{I}=\mathbf{I}^{*} \mathbf{W}^{*} \mathbf{Z}^{\prime \prime} \mathbf{W I}=(\mathbf{W I})^{*} \mathbf{Z}^{\prime \prime}(\mathbf{W I})
$$

Therefore, $\operatorname{Re}[F(j \omega)] \geq 0, \quad \forall \omega$ (condition C holds) iff the $z^{\prime}$-parameters in (28) are non-negative (this also implies $\left.z_{22}^{\prime} \geq 0, z_{33}^{\prime} \geq 0, z_{44}^{\prime} \geq 0, \cdots z_{n n}^{\prime} \geq 0\right)$. Therefore, condition C holds iff (14) holds.

In summary, conditions $\mathrm{A}, \mathrm{B}$ and $\mathrm{C}$ are necessary and sufficient for (15) or equivalently (11), which defines the $n$ port network passivity. This concludes the proof.

\section{CASE STUdY: 2-PORT AND 3-PORT NETWORKS}

In this section, we consider the special cases resulting from substituting $n=2$ and $n=3$ in Theorem 1 .

\section{A. Two-port networks}

It is easy to see that solving (20) for $n=2$ results in $u_{12}=$ $k_{12} / k_{11}, u_{12}^{*}=k_{21} / k_{11}$, and

$k_{11}^{\prime}=k_{11}, \quad k_{22}^{\prime}=\frac{k_{11} k_{22}-k_{12} k_{21}}{k_{11}}$

It is straightforward that condition (13) in Theorem 1 is same as condition (7) in the Raisbeck's criterion.

Writing $z_{i j}$ as $r_{i j}+j x_{i j}$ where $r_{i j}$ is the real part and $x_{i j}$ is the imaginary part of $z_{i j}$, we have

$$
\begin{aligned}
& {\left[\begin{array}{ll}
z_{11}^{\prime}(j \omega) & z_{12}^{\prime}(j \omega) \\
z_{21}^{\prime}(j \omega) & z_{22}^{\prime}(j \omega)
\end{array}\right]=} \\
& {\left[\begin{array}{cc}
r_{11} & \frac{1}{2}\left(r_{12}+r_{21}\right)+\frac{j}{2}\left(x_{12}-x_{21}\right) \\
\frac{1}{2}\left(r_{12}+r_{21}\right)-\frac{j}{2}\left(x_{12}-x_{21}\right) & r_{22}
\end{array}\right]}
\end{aligned}
$$

which can be diagonalized as 
$\left[\begin{array}{cc}1 & 0 \\ w_{12}^{*} & 1\end{array}\right]\left[\begin{array}{cc}z_{11}^{\prime \prime} & 0 \\ 0 & z_{22}^{\prime \prime}\end{array}\right]\left[\begin{array}{cc}1 & w_{12} \\ 0 & 1\end{array}\right]$

where

$z_{11}^{\prime \prime}=z_{11}^{\prime} \quad z_{22}^{\prime \prime}=\frac{z_{11}^{\prime} z_{22}^{\prime}-z_{12}^{\prime} z_{21}^{\prime}}{z_{11}^{\prime}}$

with

$w_{12}=z_{12}^{\prime} / z_{11}^{\prime}$ and $w_{12}^{*}=z_{21}^{\prime} / z_{11}^{\prime}$.

Using

$z_{i j}^{\prime}=\frac{1}{2}\left(z_{i j}+z_{j i}^{*}\right)$, the second condition in (31) becomes

$4 r_{11} r_{22}-\left(r_{12}+r_{21}\right)^{2}-\left(x_{12}-x_{21}\right)^{2} \geq 0$

which is same as condition (8) in the Raisbeck's criterion. Therefore, Theorem 1 is in agreement with the Raisbeck's criterion for the case where $n=2$.

\section{B. Three-port networks}

For $n=3,(20)$ is

$\left[\begin{array}{ccc}1 & 0 & 0 \\ u_{12}^{*} & 1 & 0 \\ u_{13}^{*} & u_{23}^{*} & 1\end{array}\right]\left[\begin{array}{ccc}k_{11}^{\prime \prime} & 0 & 0 \\ 0 & k_{22}^{\prime \prime} & 0 \\ 0 & 0 & k_{33}^{\prime \prime}\end{array}\right]\left[\begin{array}{ccc}1 & u_{12} & u_{13} \\ 0 & 1 & u_{23} \\ 0 & 0 & 1\end{array}\right]=$
$\left[\begin{array}{lll}k_{11} & k_{12} & k_{13} \\ k_{21} & k_{22} & k_{23} \\ k_{31} & k_{32} & k_{33}\end{array}\right]$
which can be solved to get
$k_{11}^{\prime}=k_{11}$
$k_{22}^{\prime}=\frac{k_{11} k_{22}-k_{12} k_{21}}{k_{11}}$
$k_{33}^{\prime}=\frac{k_{11} k_{33}-k_{13} k_{31}}{k_{11}}-\frac{\left(k_{11} k_{23}-k_{21} k_{13}\right)}{\left(k_{11} k_{22}-k_{12} k_{21}\right)} \frac{\left(k_{11} k_{32}-k_{31} k_{12}\right)}{k_{11}}$

Also, for $n=3$, (27) is

$\left[\begin{array}{ccc}1 & 0 & 0 \\ w_{12}^{*} & 1 & 0 \\ w_{13}^{*} & w_{23}^{*} & 1\end{array}\right]\left[\begin{array}{ccc}z_{11}^{\prime \prime} & 0 & 0 \\ 0 & z_{22}^{\prime \prime} & 0 \\ 0 & 0 & z_{33}^{\prime \prime}\end{array}\right]\left[\begin{array}{ccc}1 & w_{12} & w_{13} \\ 0 & 1 & w_{23} \\ 0 & 0 & 1\end{array}\right]=$
$\left[\begin{array}{lll}z_{11}^{\prime} & z_{12}^{\prime} & z_{13}^{\prime} \\ z_{21}^{\prime} & z_{22}^{\prime} & z_{23}^{\prime} \\ z_{31}^{\prime} & z_{32}^{\prime} & z_{33}^{\prime}\end{array}\right]$

which renders the following solution

$z_{11}^{\prime \prime}=z_{11}^{\prime}$

$z_{22}^{\prime \prime}=\frac{z_{11}^{\prime} z_{22}^{\prime}-z_{12}^{\prime} z_{21}^{\prime}}{z_{11}^{\prime}}$

$z_{33}^{\prime \prime}=\frac{z_{11}^{\prime} z_{33}^{\prime}-z_{13}^{\prime} z_{31}^{\prime}}{z_{11}^{\prime}}-\frac{\left(z_{11}^{\prime} z_{23}^{\prime}-z_{21}^{\prime} z_{13}^{\prime}\right)}{\left(z_{11}^{\prime} z_{22}^{\prime}-z_{12}^{\prime} z_{21}^{\prime}\right)} \frac{\left(z_{11}^{\prime} z_{32}^{\prime}-z_{31}^{\prime} z_{12}^{\prime}\right)}{z_{11}^{\prime}}$

As a result, a 3-port network is passive if and only if

- The $z$-parameters have no poles in the RHP

- The following residues conditions must be satisfied by the residues of the $z$-parameters at their imaginary-axis poles

$k_{i i} \geq 0 \quad i=1,2,3$

$\frac{k_{11} k_{22}-k_{12} k_{21}}{k_{11}} \geq 0$

$$
\frac{k_{11} k_{33}-k_{13} k_{31}}{k_{11}}-\frac{\left(k_{11} k_{23}-k_{21} k_{13}\right)}{\left(k_{11} k_{22}-k_{12} k_{21}\right)} \frac{\left(k_{11} k_{32}-k_{31} k_{12}\right)}{k_{11}} \geq 0
$$

${ }_{m 1}=-C_{m 1} V_{h 1}-C_{4 m 1} V_{h 1 d}+C_{6 m 1} F_{h 1}-C_{2 m 1} F_{h 1 d}$

$F_{c m 2}=-C_{m 2} V_{h 2}-C_{4 m 2} V_{h 2 d}+C_{6 m 2} F_{h 2}-C_{2 m 2} F_{h 2 d}$

$F_{c s}=-C_{s} V_{e}+C_{1} V_{e d}-C_{5} F_{e}+C_{3} F_{e d}$

where $C_{m i}=B_{m i}+\frac{K_{m i}}{s}$ and $C_{s}=B_{s}+\frac{K_{s}}{s}$ denote local PD position controllers, and $C_{6 m i}, C_{5}$ are local force feedback terms for the two masters $(i=1,2)$ and the slave, respectively. Controllers $C_{1}, C_{4 i}$ are position compensators similar to $C_{s}$ and $C_{m i}$, respectively. Lastly, $C_{2 m i}, C_{3}$ are feedforward force terms for the two masters and the slave.

In (40), $V_{h 1 d}, V_{h 2 d}, V_{e d}$ are the desired positions and $F_{h 1 d}, F_{h 2 d}, F_{e d}$ are the desired forces for the two masters and the slave, respectively. The desired velocity and force of one robot depends on the actual velocities and forces of the other two robots as the following expressions and Fig. 4 show it

$V_{h 1 d}=\alpha V_{e}+(1-\alpha) V_{h 2} \quad V_{h 2 d}=(1-\alpha) V_{e}+\alpha V_{h 1}$

$V_{e d}=\alpha V_{h 1}+(1-\alpha) V_{h 2} \quad F_{h 1 d}=\alpha F_{e}+(1-\alpha) F_{h 2}$

$F_{h 2 d}=(1-\alpha) F_{e}+\alpha F_{h 1} F_{e d}=\alpha F_{h 1}+(1-\alpha) F_{h 2}$

where $\alpha \in[0,1]$ is the weight or authority parameter specifying the relative authority that each operator has over the slave.

For perfect transparency (assuming no time delay), the choices $\quad C_{1}=Z_{s}+C_{s}, C_{2 m i}=1+C_{6 m i}, C_{3}=1+C_{5}$, $C_{4 m i}=-\left(Z_{m i}+C_{m i}\right)$ are normally made in the 4-channel architecture. For simplicity, however, let us choose $C_{3}=$ 
$C_{5}=C_{2 m 1}=C_{2 m 2}=C_{6 m 1}=C_{6 m 2}=0, C_{1}=C_{s}, C_{4 m 1}=$ $-C_{m 1}$ and $C_{4 m 2}=-C_{m 2}$. Such choices correspond to the position-error based (PEB) control architecture, which does not need any force sensor measurements.

By using (39) to (41) for the PEB controller, the impedance model of the closed-loop multilateral system is found as

$\left[\begin{array}{c}F_{h 1} \\ F_{h 2} \\ F_{e}\end{array}\right]=\left[\begin{array}{lll}Z_{11} & z_{12} & z_{13} \\ z_{21} & z_{22} & z_{23} \\ Z_{31} & z_{32} & z_{33}\end{array}\right]\left[\begin{array}{c}V_{h 1} \\ V_{h 1} \\ V_{e}\end{array}\right]$

where

$z_{11}=\left(M_{m 1} s^{2}+B_{m 1} s+K_{m 1}\right) / s$

$z_{12}=\left(-B_{m 1} s+K_{m 1} \alpha+B_{m 1} s \alpha-K_{m 1}\right) / s$

$z_{13}=\left(-B_{m 1} s \alpha-K_{m 1} \alpha\right) / s$

$z_{21}=\left(-B_{m 2} s \alpha-K_{m 2} \alpha\right) / s$

$z_{22}=\left(M_{m 2} s^{2}+B_{m 2} s+K_{m 2}\right) / s$

$z_{23}=\left(-B_{m 2} s+K_{m 2} \alpha+B_{m 2} s \alpha-K_{m 2}\right) / s$

$z_{31}=-\left(-B_{s} s \alpha-K_{s} \alpha\right) / s$

$z_{32}=-\left(-B_{s} s+K_{s} \alpha+B_{s} s \alpha-K_{s}\right) / s$

$z_{33}=-\left(M_{s} s^{2}+B_{s} s+K_{s}\right) / s$

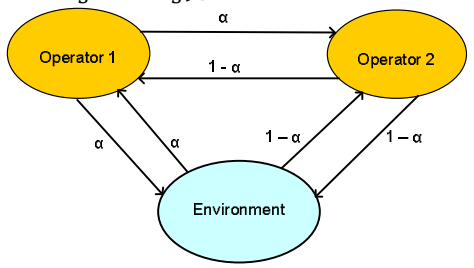

Fig. 4: A dual-user haptic teleoperation system

Evidently, (43) means that all the $z_{i j}$ have only a simple pole on the imaginary axis. Analysis of the residues $k_{i j}$ according to (13) leads to the following conditions

$k_{11}=K_{m 1} \geq 0$

$k_{22}=K_{m 2} \geq 0$

$k_{33}=-K_{s} \geq 0$

$k_{11} k_{22}-k_{12} k_{21}=B_{m 1} B_{m 2}\left(1-\alpha+\alpha^{2}\right) \geq 0$

$\frac{k_{11} k_{33}-k_{13} k_{31}}{k_{11}}-\frac{\left(k_{11} k_{23}-k_{21} k_{13}\right)}{\left(k_{11} k_{22}-k_{12} k_{21}\right)} \frac{\left(k_{11} k_{32}-k_{31} k_{12}\right)}{k_{11}} \geq 0$

The inequality (47) always holds as $1-\alpha+\alpha^{2}>0$ for all $\alpha \in[0,1]$. It turns out that the left side of (48) is equal to zero, thus the inequality (48) also holds.

Analysis of parameters $Z_{i j}^{\prime}$ according to (14) leads to

$z_{11}^{\prime}=B_{m 1} \geq 0$

$z_{22}^{\prime}=B_{m 2} \geq 0$

$z_{33}^{\prime}=-B_{s} \geq 0$

The condition $z_{11}^{\prime} z_{22}^{\prime}-z_{12}^{\prime} z_{21}^{\prime} \geq 0$ simplifies to $\omega^{2}\left(M \omega^{2}+\right.$ $N$ ) where $M$ is a function of $K_{m 1}, K_{m 2}, B_{m 1}, B_{m 2}$ and $\alpha$, and $N$ is a function of $K_{m 1}, K_{m 2}$, and $\alpha$. Therefore, both $M$ and $N$ must be non-negative such that the condition holds for all $\omega \in[0, \infty]$. We have

$N=-\left(\left(K_{m 1}+K_{m 2}\right) \alpha-K_{m 1}\right)^{2}$

which will be non-negative only when

$\alpha=K_{m 1} /\left(K_{m 1}+K_{m 2}\right)$

With $\alpha$ given in (53), the condition $N \geq 0$ leads to the following for a quadratic equation in $B_{m 1}$ (or $\left.B_{m 2}\right)$ :

$\left(K_{m 2}^{2}\right) B_{m 1}^{2}+\left(-6 B_{m 2} K_{m 1} K_{m 2}-4 B_{m 2} K_{m 2}{ }^{2}-\right.$

$\left.4 K_{m 1}{ }^{2} B_{m 2}\right) B_{m 1}+\left(B_{m 2}{ }^{2} K_{m 1}{ }^{2}\right) \leq 0$

If the equality in (54) has real solutions for $B_{m 1}$, they will be negative and the value for $B_{m 1}$ would need to be between these negative values such that the inequality in (54) holds - this is in conflict with (49). If, however, the equality in (54) has complex solutions for $B_{m 1}$, then the inequality in (54) cannot hold either. We conclude that (54) will never hold. There is no reason to check the last condition of (36).

As a result, the above PEB-controlled multilateral system is not passive. A similar fact for a PEB-controlled bilateral teleoperation system (2-port network case) is well known (such a system is neither passive nor absolutely stable).

\section{Conclusions}

A new criterion for passivity analysis of multi-user haptic systems was proposed. The proposed criterion is based on the analysis of immittance parameters of the $n$-port network of the multilateral haptic system. Theorem 1 gives the necessary and sufficient conditions that the immitance parameters and their residues must satisfy for the $n$-port network to be passive. It was shown that for $n$-port networks, $n$ conditions for the residues plus $n$ conditions for the immitance parameters must be satisfied. The theorem was applied to the case $n=2$, resulting in the same conditions as in the Raisbeck's criterion for passivity of two-port networks. Then, the theorem was used to draw the necessary and sufficient conditions for passivity of three-port networks. Finally, the theorem was applied to a dual-user haptic teleoperation system. Although passivity is a good tool for investigating the stability of multilateral haptic systems where the operators and/or environments are unknown, its results are conservative compared to those from absolute stability. Future work will be directed towards finding the necessary and sufficient conditions for absolute stability of an $n$-port network.

\section{REFERENCES}

[1] M. Tavakoli, A. Aziminejad, R.V. Patel, M. Moallem, "High-Fidelity Bilateral Teleoperation Systems and the Effect of Multimodal Haptics," IEEE T. Sys. Man Cyb. - Part B, vol. 37, no. 6, pp. 1512-1528, 2007.

[2] B. Hannaford, "A design framework for teleoperators with kinaesthetic feedback,” IEEE T. Robotics \& Aut., vol. 5, no. 4, pp. 426-434, 1989.

[3] David J. Bennett, "What are the advantages of variable stiffness control?" IEEE Engineering in Medicine and Biology, pp. 86-87, 1992.

[4] Y. Matsuoka and R. D. Howe, "Hand impedance change during learning of a novel contact task," World Congress on Medical Physics and Biomedical Engineering, 2000.

[5] S. Sirouspour and P. Setoodeh, "Adaptive nonlinear teleoperation control in multi-master/multi-slave environments," in the Proc. IEEE Int. Conf. Control Applications, Aug. 2005, pp. 1263-1268.

[6] C. R. Carignan and P. A. Olsson, "Cooperative control of virtual objects over the internet using force-reflecting master arms," in Proc. of IEEE Int. Conf. on Rob. And Auto., 2004, vol. 2, pp. 1221-1226.

[7] B. Chebbi, D. Lazaroff, et al., "Design and implementation of a collaborative virtual haptic surgical training system," Proc. IEEE Int. Conf. Mech. Auto., 2005, pp. 315-320.

[8] D. Feth et al., "Shared-Control Paradigms in Multi-Operator-SingleRobot Teleoperation," Human Centered Robot Sys., pp. 53-62, 2009.

[9] S. Sirouspour, "Robust Control Design for Cooperative Teleoperation," Proceedings of the 2005 IEEE International Conference on Robotics and Automation, pp 1133 -1138, Spain, April 2005.

[10] R. J. Anderson, M. W. Spong, "Bilateral control of teleoperators with time delay," IEEE T. Aut. Control, vol. 34, no. 5, pp. 494-501, 1989.

[11] S. Haykin, Active Network Theory. Addison-Wesley, 1970.

[12] G. Lombardi, B. Neri, "Criteria for the evaluation of unconditional stability of microwave linear two-ports: a critical review and new proof," IEEE T. Micro. Theory Tech., vol. 47, no. 6, pp. 746-751, 1999.

[13] E.L. Tan, X. Sun, and K.S. Ang, "Unconditional Stability Criteria for Microwave Networks," PIERS Proceedings, Beijing, China, 2009.

[14] B. Khademian and K. Hashtrudi-Zaad, "A four-channel multilateral shared control architecture for dual-user teleoperation systems," Int. Conf. Intelligent Robots and Systems, pp. 2660-2666, 2007. 